\title{
Strategies for Successful Drug-Release in Nano Medicine: An Overview
}

\author{
Jean Felix Mukerabigwi ${ }^{1,2 *}$ \\ ${ }^{1}$ Department of Polymer Science, Engineering, University of Science and Technology of China, China \\ ${ }^{2}$ Chemistry Department, University of Rwanda, Rwanda \\ *Corresponding author: Jean Felix Mukerabigwi, Department of Polymer Science and Engineering, University of Science and Technology of China, \\ China
}

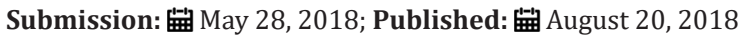

\begin{abstract}
Since the introduction of nanotechnology into biomedicine, there have been myriads of successful applications of nano materials into treatment of various diseases. Among them, the utilization of nano materials in drug delivery system (DDS) to enhance the pharmacological effects has been the most extensively studied to date. Particularly, the targeted drug delivery and release has been the hot subject of many researches in the field of current modern nano medicine. In this mini review, we briefly overview the currently applied approaches to achieve drug successful release for enhanced bioavailability at the targeted treatment site.
\end{abstract}

Keywords: Drug delivery; Stimuli responsive; Nano materials; Nano technology; Biomedicine

Abbreviations: DDS: Drug Delivery System; EPR: Permeability Retention Effect; ROS: Reactive Oxygen Species

\section{Introduction}

Remarkably, the application of nanotechnology has witnessed a tremendous progress into modern medicine with the aim to enhance the therapeutic effect of the standard drugs for treatment of various diseases and genetic disorders, thereby developing new treatment concepts. In fact, given that most of the small molecule drugs are associated with some limitations during treatment such as poor targeting ability, undesirable accumulation in normal tissues/organs which often cause damage to normal tissues, unappreciated half-lives during blood circulation, poor solubility in most aqueous solvents, etc.,-there has been a growing interest to design various strategies to improve the treatment efficacies of those small molecule drugs and by simultaneously impeding the systemic toxicity in the patients [1].

Among the most studied strategies, the following two approaches hold great promises in the future medicine to enhance the clinical effect during treatment of various diseases, these are:

1. The development of prodrugs which can preferably remain inactive during the circulation and only become active at the target in the presence of proper conditions [2].

2. The engineering of nano materials which can be exploited as the vehicles to transport these small molecule drugs to the treatment site for better clinical effects in what is commonly known as Drug-Delivery System (DDS).
In this respect, various nano materials from either organic or inorganic sources and with different architectures such as micelles, vesicles, dendrimers, etc., have been extensively studied and applied in DDS [3]. For example in chemotherapy, large molecules with a diameter size from tenth to several hundredth nanometers are prone to accumulate in tumor microenvironment due to the enhanced permeability and retention effect (EPR) $[4,5]$.

Moreover, nano carrier systems can also be used to safely deliver fragile and active therapeutic biomaterials such as exogenous therapeutic enzymes without being inactivated or destroyed by the immune system within the body. What is impressing about the above two highlighted emerging strategies is that either prodrugs or these drug nano vehicles can be further functionalized and modified with targeting ligands on their surface for tissue or cellspecific delivery to enhance their targeting ability and minimize side-effects. In this fashion, passive or active targeting strategies can be utilized to deliver the therapeutic agents to the specific diseased organ or tissue [6].

Therefore, once these prodrugs or engineered drug nano vehicles reach the desired destinations, it is very important to ensure that the parental or loaded drug materials can be freely released from their host platforms for enhanced drug uptake in the tissues of interest during treatment. In this respect, various types 
of triggering moieties have so far been proposed and studied for successful drug release. In this report, we concisely overview the current and prominent approaches developed to locally induce the drug-release on-demand in modern nano medicine (Figure 1).

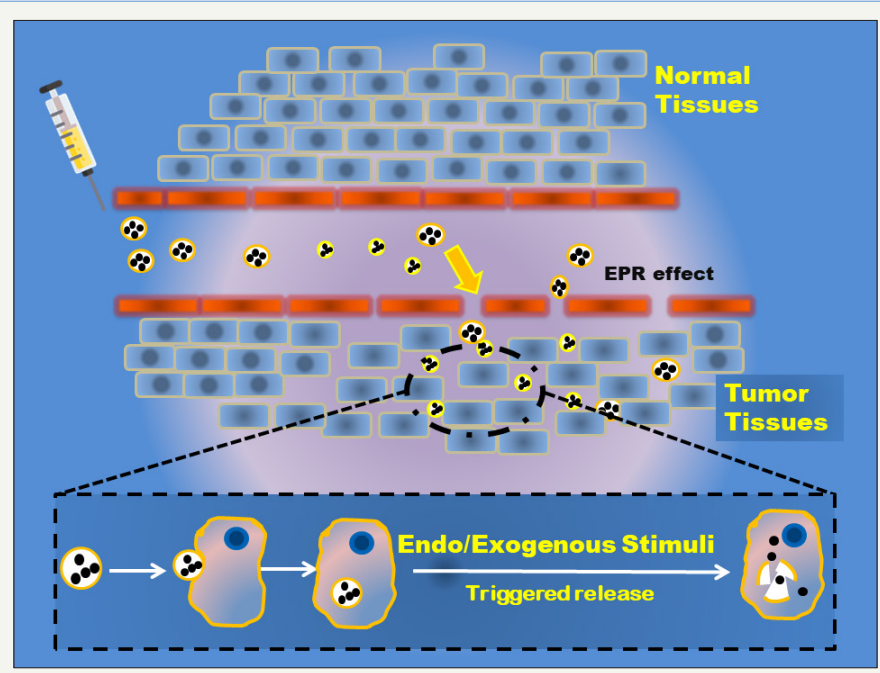

Figure 1 : Schematic illustration of a proposed mechanism for how drug Nano Carriers can enter the Tumour microenvironment after intravenous administration through leaky endothelial tissue (EPR effect), and then be internalized by Tumour cells through passive means. Once they reach in the treatment cells, they can be activated either by internal or external triggering agents to successfully release their therapeutic cargoes for treatment.

\section{Approaches Applied to Induce Drug Release}

Table 1: Summary of general advantages and limitations of various strategies used to induce drug release in nanomedicine

\begin{tabular}{|c|c|c|}
\hline Source of Triggering Stimuli & Advantage & Limitation \\
\hline $\begin{array}{l}\text { Endogenous Stimuli } \\
\text { (Eg.: pH, redox potential, temperature, enzyme, hypox- } \\
\text { ia, etc.) }\end{array}$ & $\begin{array}{c}\text { Achieve high bioavailability; } \\
\text { High specificity; } \\
\text { High safety; } \\
\text { Achieve locally intrinsic } \\
\text { drug release on demand. }\end{array}$ & $\begin{array}{l}\text { Less controllable drug release; } \\
\text { Less applicability. }\end{array}$ \\
\hline $\begin{array}{c}\text { Exogenous Stimuli } \\
\text { (Eg : light, ultrasound, magnetic field, heat, etc.) }\end{array}$ & $\begin{array}{l}\text { Feasible to achieve spatial controllable } \\
\text { drug release; } \\
\text { Applicable to many pathological con- } \\
\text { ditions. }\end{array}$ & $\begin{array}{l}\text { Neighboring normal tissues can be affected during } \\
\text { treatment } \\
\text { Stimuli can be interfered by many external param- } \\
\text { eters. (eg: tissue thickness, etc.) }\end{array}$ \\
\hline
\end{tabular}

Herein, we roughly summarize these approaches into two groups depending on the sources and locations of the triggering agents to induce drug-release for treatment and therapeutic application in biomedicine (Table 1).

\section{Endogenous stimuli}

With greater understanding of the difference between normal and pathological tissues for example, the gradients between the intracellular and extracellular environments of normal and abnormal tissues, this enabled the researchers to develop internal stimuli responsive nano Carriers which can solely release their cargo when they reach to the desired treatment site without harming normal cells [7]. In this scenario, stimuli responsive nano Materials for therapeutic applications can be constructed to respond to internal stimuli such as $\mathrm{pH}$, local intracellular triggering enzymes, temperature, intracellular redox potentials (such as glutathione (GSH)), or reactive oxygen species (ROS) such as (hydrogen peroxide $\left(\mathrm{H}_{2} \mathrm{O}_{2}\right.$, free radicals, etc), hypoxia, etc. Particularly, it has been reported that there is a high secretion of redox potentials and ROS in tumor tissues than in normal tissues, moreover, the tumor micro-environments tend to be more acidic compared to the normal physiological environment in biological system. Moreover, some pathological conditions such as inflamed tissues have been reported to possess high temperature than normal physiological conditions [8].

This strategy which involves taking advantage of endogenous stimuli to trigger drug release can offer ultimate target specificity 
and release on demand locally. Moreover, prolonged drug release can be achieved to increase the drug bioavailability, enhanced pharmacological effects and minimized unnecessary toxicities. However, only limited pathological conditions are applicable for internal stimuli responsive nano Carriers. Therefore, further means of release have also been developed such as the application of external triggering agents as discussed in the next subsection.

\section{Exogenous stimuli}

The Nano Carriers can be purposely designed to respond to external stimuli. In this case, various external stimuli such as ultrasound, light (irradiation), magnetic field, heat, etc., have been used to trigger the drug-release at the diseased site [9]. Compared to endogenous stimuli responsive systems, the exogenous stimuli responsive systems could intrinsically or introduce contrast agents to visualize the accumulation of Nano Particles in the target tissues, cells or organelles and then activate the Nano Carriers out of body for example by irradiation, magnetic field, heat, ultrasound or other triggers at desired time [10]. Thus, it is possible to spatially control the release simply by turning on/off the stimuli source. However, several external parameters such as the tissues location or thickness, and so on can sometimes interfere and prevent to reach the maximized therapeutic outcome.

\section{Conclusion and Future Perspectives}

In summary, nanotechnology hold great advantage in modern Nano Medicine as it can make it feasible to achieve targeted drug delivery at the treatment site to enhance drug bioavailability and improved clinical effects as well as the reduced undesired systemic toxicities. However, though there are unnumbered ways to construct Nano Carriers for drug delivery, their proposed means for drug release is still limited and not applicable for most of diseased sites in various pathological conditions. Therefore, there is still an empty room to be covered by the future researchers while developing more novel and sophisticated drug release strategies.

\section{References}

1. Ganta S, Devalapally H, Shahiwala A, Amiji M (2008) A review of stimuli-responsive nano carriers for drug and gene delivery. Journal of Controlled Release 126(3): 187-204.

2. Rautio J, Kumpulainen H, Heimbach T, Oliyai R, Järvinen T, et al. (2008) Prodrugs: design and clinical applications. Nature Reviews Drug Discovery 7(3): 255-270.

3. Cabane E, Zhang X, Langowska K, Palivan CG, Meier W (2012) Stimuli Responsive Polymers and Their Applications in Nanomedicine. Biointerphases 7(1): 9.

4. Iyer AK, Khaled G, Fang J, Maeda H (2006) Exploiting the enhanced permeability and retention effect for tumor targeting. Drug Discovery Today 11(17): 812-818.

5. Greish K (2010) Enhanced Permeability and Retention (EPR) Effect for Anticancer Nanomedicine Drug Targeting, in: S.R. Grobmyer, BM Moudgil (Eds.) Cancer Nanotechnology: Methods and Protocols Humana Press Totowa, NJ, USA, pp. 25-37.

6. Nicolas J, Mura S, Brambilla D, Mackiewicz N, Couvreur P (2013) Design functionalization strategies and biomedical applications of targeted biodegradable/biocompatible polymer based nano carriers for drug delivery. Chemical Society Reviews 42(3): 1147-1235.

7. Wang S, Huang P, Chen X (2016) Stimuli-Responsive Programmed Specific Targeting in Nanomedicine. ACS nano 10(3): 2991-2994.

8. Yiting $Q$, Jianqin $W$, Liqian Z, Wen M, Yuanyuan Y, et al. (2018) Stimuliresponsive nano therapeutics for precision drug delivery and cancer therapy. Wiley Interdisciplinary Reviews Nanomedicine and Nano biotechnology e1527.

9. Wang Y, Kohane DS (2017) External triggering and triggered targeting strategies for drug delivery. Nature Reviews Materials 2(6): 17020.

10. Yao J, Feng J, Chen J (2016) External-stimuli responsive systems for cancer theranostic. Asian Journal of Pharmaceutical Sciences 11(5): 585-595.
Creative Commons Attribution 4.0 International License

For possible submissions Click Here

\section{Submit Article}

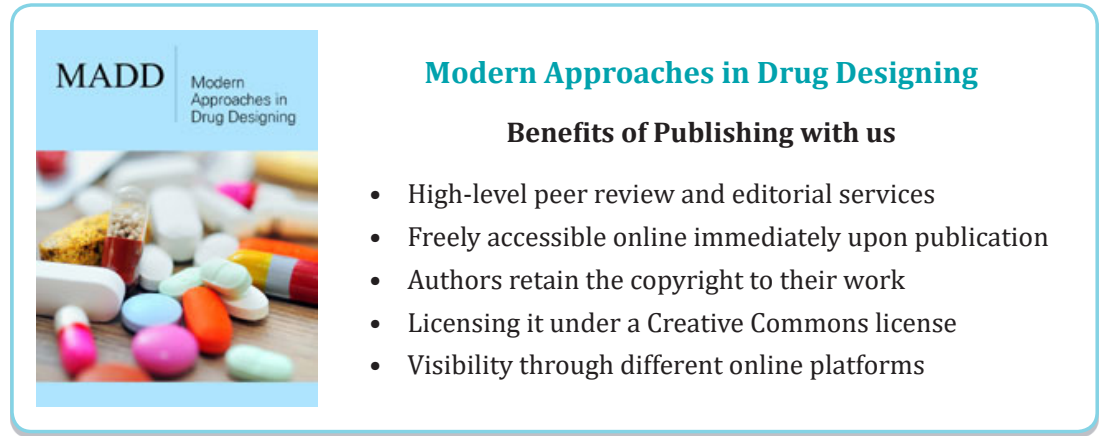

